\title{
ON TWO-DIMENSIONAL CONFORMAL INVARIANCE AND THE FIELD THEORY OF STRINGS
}

\author{
D. FRIEDAN \\ Enrico Fermi Institute and Department of Physics, University of Chicago, \\ Chicago, IL 60637, USA
}

Received 1 August 1985

\begin{abstract}
An algebraic approach to string field theory is proposed. The string field is written as the quantum stress-energy tensor of two-dimensional nonlinear field theory. The equation of motion is the condition of two-dimensional conformal invariance, expressed as a quadratic operator product relation. The gauge structure of the linearized equation of motion is analyzed. Some of the remaining problems are discussed.
\end{abstract}

A clue to the structure of string theory is given by the renormalization group equation of the general nonlinear model in two dimensions $[1,2]$. The nonlinear model consists of a field $x^{\mu}(\xi)$ which is a function from points $\xi=\left(\xi^{1}, \xi^{2}\right)$ on the plane to a $d$-dimensional manifold, call it spacetime. The action of $x^{\mu}(\xi)$ is

$S(x)=\frac{1}{4 \pi} \int \mathrm{d}^{2} \xi g_{\mu \nu}(x) \partial_{a} x^{\mu} \partial_{a} x^{\nu}$

The coupling is given by a space time metric $g_{\mu \nu}(x)$. The renormalization group (RG) equation, expanded in powers of $\left(g_{\mu \nu}\right)^{-1}$, is

$\Lambda \frac{\partial}{\partial \Lambda} g_{\mu \nu}=R_{\mu \nu}+\frac{1}{2} R_{\mu \rho \sigma \tau} R_{\nu} \rho \sigma \tau+\ldots$,

where $\Lambda^{-1}$ is a distance scale on the plane. The RG equation is covariant under spacetime reparame trizations, to all orders in the perturbative expansion. The crucial point is that the RG fixed point equation in a two-dimensional quantum field theory (2D QFT) gives a generally covariant equation of motion for gravity. In the long distance limit on spacetime, $\left(g_{\mu \nu}\right)^{-1} \rightarrow 0$, the fixed points become the solutions of Einstein's equation $R_{\mu \nu}=0$.

For a string theory to be a complete theory of

is This work was supported in part by US Department of Energy grant DE-FG02-84ER-45144 and the Alfred P. Sloan Foundation. physics [3], the string field must describe the environment in which string moves. It seems reasonable to suppose that the string field is exactly this description. The dynamics of string is described by a sum over world surfaces, i.e. a 2D QFT [4]. For example, eq. (1) can be interpreted as the action of the parametrized world surface of a string moving in a curved spacetime with metric $g_{\mu \nu}(x)$. The metric coupling describes the environment, as a component of the string field. The complete description of the environment of a string is the general 2D QFT, with all possible local self-interactions of the world surface $x^{\mu}(\xi)$. The coupling describing the self-interactions are tensors on spacetime, for example $t_{\mu_{1} \ldots \mu_{k}}(x) \times$ $\partial_{a_{1}} x^{\mu 1} \ldots \partial_{a_{k}} x^{\mu k}$ is a self-interaction of naive dimension $k$.

A 2D QFT gives consistent dual Born amplitudes for string scattering only if it is conformally invariant ${ }^{\neq 1}$ and scale invariance, the RG fixed point condition, is equivalent to conformal invariance in a local QFT $\neq 2$ [13]. The same techniques which show that eq. (2) is invariant under general coordinate transformations

\#1 This is described from the point of view of $2 D$ QFT in ref. [5], see also ref. [6].

\$2 The connection between refs. $[1,2]$ and $[5,6]$ was discussed in the period 1983-84 by, among others, Friedan, Lovelace, Shenker, and Witten. The author was interested in the connection by Bardacki [7]. Recent work on conformal invariance and string theory includes refs. [8-12]. 
of spacetime [2], can be applied to the completely general nonlinear model to show that the RG fixed point equation gives a generally covariant equation of motion for the string field.

In order to find the long distance effective field theory of string, it is useful to describe the 2D QFT by its classical action, because the RG decouples (at long distance) the nonrenormalizable parts of the 2D action (massive string modes) from the renormalizable parts (massless string modes), leaving a classical long distance equation of motion on the relevant modes, e.g. $R_{\mu \nu}=0$, whose solutions do not excite irrelevant modes [13]. But the classical 2D action is not a useful description for string field theory. The dynamics must be nonlocal in the tensor fields in order to accomplish the long distance decoupling. The RG equation is not uniquely defined, since it depends on the choice of renormalization scheme. There is no sign of the string-like structure expected in a string field equation. The interaction term in the string field equation should be bilinear in the field, representing the joining and splitting of string. Finally, the only obvious symmetry of the fixed point equation is spacetime coordinate invariance. String field theory should have a much larger gauge invariance.

The RG fixed point equation is equivalent to $T_{a a}(\xi)=0$, where the 2D stress-energy tensor $T_{a a}$ is written as a functional of the $2 \mathrm{D}$ action. In complex tensor notation $\left(z=\xi^{1}+\mathrm{i} \xi^{2}\right), T_{z \bar{z}}=0$. Conformal invariance can also be expressed as the singular operator product expansion [6]:

$$
\begin{aligned}
& T_{z z} T_{w w} \approx(z-w)^{-4} c / 2+(z-w)^{-2} 2 T_{w w} \\
& \quad+(z-w)^{-1} \partial_{w} T_{w w}+\ldots
\end{aligned}
$$

$T_{z z} \bar{T}_{\bar{w} \bar{w}} \approx 0+\ldots$,

where $c=d$. This version of the 2D conformal invariance condition, using only linear and bilinear expressions in $T_{z z}$, seems a promising source for the string field equation.

To begin investigating the structure of these equations, consider the simpler case of the open string. $T_{z z}=T(z)$ describes the environment on the boundary $\operatorname{Im}(z)=0$ of the open string, and eq. (3) is the conformal invariance condition. Choose a background conformal field theory with stress-energy tensor $T_{0}(z)=\Sigma_{n=-\infty}^{\infty} z^{-n-2} L_{n}$. Eq. (3) is equivalent to the Virasoro algebra commutation relations

$$
\left[L_{m}, L_{n}\right]=(m-n) L_{n+n}+\frac{1}{\Gamma 2} c\left(m^{3}-m\right) \delta_{m+n, 0},
$$

with $c=d$. Expand $T(z)$ around the background, $T(z)=T_{0}(z)+\phi(z)$ with $\phi(z)$ a translation covariant quantum field in the QFT described by $T_{0}(z)$ :

$\phi(z)=\mathrm{e}^{z L-1} \phi(0) \mathrm{e}^{-z L-1}$.

This amounts to allowing only translation invariant nonlinear models, that is models whose couplings have no explicit $\xi$ dependence. Then eqs. (3), (4) can be rewritten,

$$
\begin{gathered}
T_{0}(z) \phi(0)+\phi(z) T_{0}(0)+\phi(x) \phi(0) \\
\approx z^{-2} 2 \phi(0)+z^{-1} \partial \phi(0) .
\end{gathered}
$$

To get an operator representation of the fields, perform a radial quantization with the background $L_{0}$ as hamiltonian. The radial quantization gives a one to one correspondence between the quantum field $\phi(z)$ and the Hilbert space state $|\phi\rangle=\phi(0)|0\rangle$, where $|0\rangle$ is the $\mathrm{SL}_{2}$ invariant ground state of the background $2 \mathrm{D}$ QFT, satisfying $L_{n}|0\rangle=0, n \geqslant-1$. Let $L_{n} \phi(z)$ be the translation covariant quantum field corresponding to the state $L_{n} \phi(0)|0\rangle$. Then

$T_{0}(z) \phi(w) \approx \sum_{n=-1}^{\infty}(z-w)^{-n-2} L_{n} \phi(w)$.

Use the correspondence between states and $2 \mathrm{D}$ quantum fields to put eq. (7) in the form

$$
\begin{aligned}
& \sum_{n=-1}^{\infty} z^{-n-2}\left[\left(L_{n}-\delta_{n,-1} L_{-1}-2 \delta_{n, 0}\right)\left|\phi^{\circ}\right\rangle\right. \\
& \left.+(-1)^{n} L_{n} \phi(z)|0\rangle\right]+\phi(z)|\phi\rangle \approx 0 .
\end{aligned}
$$

Note that the string fields have now been identified with the states of the first quantized string.

Now consider the linearized equation of motion. Expanded in powers of $z$, it is $(n \geqslant-1)$

$$
\begin{aligned}
0 & =\left(L_{n}+\sum_{k=0}^{\infty} \frac{(-1)^{n+k}}{k !} L_{-1}^{k} L_{n+k}\right. \\
& \left.-L_{-1} \delta_{-1, n}-2 \delta_{0, n}\right)|\phi\rangle .
\end{aligned}
$$

After some algebra - applying Virasoro operators to eq. (10), taking linear combinations of the resulting 
equations for different $n$, and using the fact that

$L_{-1}|\chi\rangle=0$ implies $|\chi\rangle=0$ - eq. (10) becomes

$L_{n}|\phi\rangle=L_{1}\left[\left(L_{0}-1\right)^{-1} L_{1}\right]^{n-1}|\phi\rangle \quad(n \geqslant 2)$,

$0=L_{1} \frac{1}{2}(1+\tau)|\phi\rangle$,

$0=\left(L_{0}-1\right) \frac{1}{2}(1+\tau)|\phi\rangle$.

The operator

$\tau=\sum_{n=0}^{\infty} \frac{(-1)^{n}}{n !} L_{-1}^{n}\left[L_{1}\left(L_{0}-1\right)^{-1}\right]^{n}$

is the twist operator in the universally envelopping algebra of $\mathrm{SL}_{2}: \tau^{2}=1$, and $\tau L_{n}=(-1)^{n} L_{n} \tau$ for $n=$ $-1,0,1$.

The projection condition $\tau|\epsilon\rangle=|\epsilon\rangle$ is equivalent to $L_{1}^{2 m+1}|\epsilon\rangle=\sum_{k=0}^{\infty} p_{k} L_{-1}^{2 k+1}$

$$
\times\left[L_{1}\left(L_{0}-1\right)^{-1}\right]^{2 k+1} L_{1}^{2 m+1}|\epsilon\rangle \quad(m \geqslant 0)
$$

(actually $m=0$ alone is also equivalent). The equation $\left(\tau L_{1}+L_{1} \tau\right)|\epsilon\rangle=0$ then leads to a recursion relation,

$p_{0}=1 / 2$,

$p_{n}=A_{n+1 / 2}(s)-\sum_{k=1}^{n} A_{k}(s) p_{n-k}$,

$A_{k}(s)=\frac{1}{2} \frac{\Gamma(2 s+1) \Gamma(s+2 k)}{\Gamma(2 s+2 k) \Gamma(s+1)} \frac{1}{(2 k !)}$,

which gives the same series $p_{n}$ whatever the value of $s$. The recursion relation is used to show the equivalence between eq. (10) and eqs. (11)-(13).

Eq. (10) is local, i.e. polynomial in the $L_{n}$ for any regular state $|\phi\rangle$ (killed by all $L_{n}$ for $n$ large enough). Eqs. (11)-(13) are local everywhere except at the singular points $L_{0}=2,3,4, \ldots$. The equivalence is between solutions of (10) and solutions of (11)-(13) which continue analy tically from the nonsingular region. Alternatively, eqs. (11)-(13) should be multiplied by analytic functions of $L_{0}$ with the minimal set of zeros needed to remove the singularities.

Eq. (13) can be interpreted as a conventional equation of motion, and eqs. (11), (12) can be interpreted as conventional gauge conditions. The gauge, however, is not completely fixed. The linearized gauge genera- tors are the pure gauge solutions of (11)-(13). The pure gauge solutions of eqs. (12), (13) are $|\phi\rangle=$ $L_{-1}|\epsilon\rangle$ with $\tau|\epsilon\rangle=|\epsilon\rangle$, i.e. eq. (15). The gauge conditions (11) fix

$$
\begin{aligned}
& L_{n}|\epsilon\rangle=\frac{n+1}{n+2}\left(2 L_{0}+n\right)\left[L_{1}\left(L_{0}-1\right)^{-1}\right]^{n}|\epsilon\rangle \\
& +\left(L_{0}+n+1\right) \\
& \quad \times \sum_{k=1}^{\infty} \frac{(-1)^{k}(n+1) ! L_{-1}^{k}\left[L_{1}\left(L_{0}-1\right)^{-1}\right]^{k+n}|\epsilon\rangle}{(n+k-1) !(n+k+1)(n+k+2)} \\
& (n \geqslant 1),
\end{aligned}
$$

which is consistent with eq. (15).

On reach of the infinitely many levels of Hilbert space there are gauge generators. For example, any state satisfying all the usual gauge conditions $L_{n}|\epsilon\rangle=$ $0, n \geqslant 1$ gives a gauge invariance of eqs. (11)-(13).

When $T_{0}(z)$ is the flat spacetime background, the lowest level gauge generators are $|\epsilon\rangle=|p\rangle$ where $|p\rangle$ is the ground state of energy-momentum $p^{\mu}$. These are the gauge generators of the low energy effective gauge theory of the open string. For the closed string the corresponding generators would be the spacetime reparame trizations.

The additional gauge condition $L_{1}|\phi\rangle=0$ produces the standard completely gauge fixed free theory with inverse propagator $L_{0}-1$.

Note that this approach to string field theory would also lead to a new formulation of the theory of critical phenomena. The nongauge solutions of eqs. (11)-(13) correspond to the marginal operators of critical phenomena. The gauge generators correspond to the redundant operators $[14] \neq 3$.

For supersymmetric strings $[11,15,16]$ the analog of conformal invariance ${ }^{\neq 4}$ is the condition of twodimensional superconformal invariance, expressed as an operator product condition on the super stressenergy tensor $[10,17]$. Every superconformal field theory can be enlarged by introducing the spin fields [10]. These are the local quantum fields which are double valued in the neighborhood of any fermionic

\footnotetext{
${ }^{\$ 3}$ I thank S. Shenker for pointing out the redundant operators and ref. [14].

$\neq 4$ Recent work on 2D superconformal invariance in superstring theory includes refs. [9-11,13].
} 
field. The enlarged theory is not strictly speaking a quantum field theory, because of the doubling. The spin model is the local QFT which results from projecting on the sector of even fermion number, $(-1)^{F}=$ 1. This construction generalizes to nonconformal QFTs for which $(-1)^{F}$ is defined and conserved. These are the 2D QFTs which correspond to the string fields of the supersymmetric string. For type II superstrings the 2D supersymmetry is $N=1$; for heterotic strings it is $N=1 / 2$. Eq. (2) has an analogue for supersymmetric nonlinear models [18], the crucial difference being that the RHS vanishes to all orders if $R_{\mu \nu}=0$, at least if the spacetime is Kähler [19], and for the heterotic case if the background gauge field is the metric connection.

Clearly this appproach to string field theory is not finished. The above calculations might be thought of as a feasibility study for the idea that the commutation relations of the 2D stress-energy tensor could provide a gauge invariant string field equation with a bilinear interaction term. There are many problems remaining. The gauge algebra found above is too small. The closed string and the interaction must be investigated. Note that eq. (3) is written in the Heisenberg picture associated with $T_{a b}$. But gauge fixed calculations are done in the Heisenberg picture of the background 2D QFT. There must be an covariance under change of picture. There is the difficulty that the bilinear term in eq. (9) is not restricted to integer powers of $z$. The bilinear term in eq. (9) is closely related to the overlap integral which gives the usual string vertex. To see this perform the Klein transformation $w=\alpha \log (1-z)+(1-\alpha) \log (z)$, which takes the upper half plane to the split strip. The operator product of conformal fields is taken to the overlap integral of the corresponding states. The complicated algebraic manipulations leading to eqs. (11)(13) need to be rationalized. The use of only the traceless part of the stress-energy tensor in the equation of motion seems artificial, and it must be shown how to construct the rest of the stress-energy tensor off-shell. Finally, there is as yet no action principle for the conformal invariance equations. It is not apparent that the RG generator is the gradient of an action functional.

The first quantized theory given by the string field as background field only has the duality property when the string field is on-shell. There must be a more general invariance in the off-shell string fields. Translation invariance on the surface is clearly not a natural condition, which presumably explains the limited amount of gauge invariance in eqs. (11)-(13). The environment in which a string moves is not a QFT on any particular surface. In fact, the RG equation does not see the global structure of the surface; the infinitesmal RG transformation acts locally on the surface.

All that is needed to describe the motion of a string is a description of infinitesimal motion, because finite motion can be described by patching infinitesimal surface elements together with reparametrizations. A reparametrization covariant $Q F T$ is a $Q F T$ on an arbitrarily small piece of parametrized surface, along with a stress-energy tensor which generates infinitesmal reparametrizations. The QFT is not necessarily translation invariant the stress-energy tensor is not necessarily conserved.

Dirac [20] gave a language for quantum field theory which is well-suited to reparametrization covariant QFT. Correlations between regions of the surface are mediated by wave functions on the boundaries. Each closed curve $\mathrm{C}$ is associated with a space of states $L_{2}[C]$, e.g. $L_{2}$ of maps from $C$ to space time. A QFT assigns to each curve $C$ a state $|0\rangle_{C}$ in $L_{2}[C]$, i.e. the functional in tegral over $x^{\mu}(\xi)$ inside $C$, depending on the boundary value $x^{\mu}(s)$ on C. There is a composition law taking $\mathrm{L}_{2}\left[\mathrm{C}_{1}\right] \times$ $\mathrm{L}_{2}\left[\mathrm{C}_{2}\right] \rightarrow \mathrm{L}_{2}\left[\mathrm{C}_{3}\right]$ whenever $\mathrm{C}_{1} \cup \mathrm{C}_{2}=\mathrm{C}_{3}$, i.e. the functional integral over the common boundary. The states $|0\rangle_{C}$ are closed under composition.

Define $L_{2}[\xi]$ and $|0\rangle$ e as the limits of $L_{2}[C]$ and $|0\rangle_{C}$ as $C$ contracts to $\xi$. The QFT is completely determined by the $|0\rangle \xi$. Local fields can be identified with functions $\phi(\xi)$ ín $\mathrm{L}_{2}[\xi]$. Operator realizations are given by radial quantizations, using systems of concentric circles (around arbitrary points $\xi$ ).

The propagation of information in reparame trization covariant 2D QFT parallels the propagation of information on the string, if the state of a string is associated not with a global parame trization of the string, but with a covering of the string by infinitesmal parameter patches.

The stress-energy tensor acts at boundaries by the linear operator

$$
\oint_{\mathrm{C}} \mathrm{d} \xi^{a} \hat{T}_{a b}(\xi) v^{b}(\xi): \mathrm{L}_{2}[\mathrm{C}] \rightarrow \mathrm{L}_{2}\left[\mathrm{C}^{\prime}\right]
$$


where the reparametrization $\xi^{a} \rightarrow \xi^{a}+v^{a}(\xi)$ takes the curve $\mathrm{C}$ to $\mathrm{C}^{i}$, and $\hat{T}_{a b}=\epsilon_{a}^{c} T_{c b}$. The transform of $|0\rangle_{C}$ is not equal to $|0\rangle_{C^{\prime}}$, which is to say that the 2D QFT is not reparametrization invariant.

On any surface covered by infinitesimal parameter patches, the reparame trization covariant QFT defines a QFT on each patch, and the reparametrization generators for the infinitesimal transition functions can be used to glue the local QFTs together. Reparametrizations give different but equivalent QFTs. This picture - systems of parameters and systems of local QFTs - is derived from the systems of coordinates construct defined in ref. [2].

A reparame trization covariant QFT can be thought of as a connection for the infinitesimal local reparametrizations (germs of reparametrizations) in the local bundle of Hilbert spaces $L_{2}[\xi]$. Conformal invariance means that the connection has no torsion for the local analytic reparametrizations. Then the reparametrization covariant QFT defined on an infinitesimal piece of surface extends naturally to a globally defined conformal field theory on any Riemann surface, because the patching reparametrizations of a Riemann surface are locally analytic.

The local conformal transformations at $z_{0}, z \rightarrow$ $z+\epsilon_{n}\left(z-z_{0}\right)^{n+1}, n \geqslant-1$, are generated by operators $L_{n}\left(\xi_{0}\right)$ acting on $L_{2}\left[\xi_{0}\right]$. Conformal invariance is the condition $L_{n}(\xi)|0\rangle_{\xi}=0, n \geqslant-1$, which again is bilinear in form. Since the reparametrization covariant QGT is defined on an arbitrarily small piece of surface, the $\xi$ dependence can be represented by formal power series around $\xi=0$.

An especially interesting mode is the dilaton zero mode, which is the string coupling constant. In the nonlinear model the dilaton zero mode only enters the boundary interaction, in the form

$$
\oint_{\mathrm{C}} \mathrm{d} s \kappa(s) \Phi(x(s s)),
$$

$\kappa(s)$ being the geodesic curvature density on the boundary $\mathrm{C}$. The corresponding mode in the reparametrization covariant QFT is possibly the density $|0\rangle_{\xi}$ modulo total divergences.

Other attempts to construct gauge invariant string field theories [21-26] start from the known gauge fixed flat space theory, then add fields and interactions by hand to achieve gauge invariance. Sections 1-7 of ref. [24] present a somewhat different ap- proach. There the attempt is to construct the theory by bootstrap methods assuming only gauge invariance and spacetime locality. But in none of these attempts is there suggested an underlying role for conformal invariance. The role of conformal invariance must be understood, because in these approaches the background is a conformally invariant QFT. The Virasoro operators and the overlap integral of that background conformal QFT are used to write the action functional of the string field. When the background is shifted (staying on-shell), the string field must appear in the new Virasoro algebra and overlap in tegral.

The present approach is an attempt to realize some of the suggestions in sections 10 and 11 of ref. [24]. The string field as stress-energy tensor is a variation on the idea of the ground state of the 2D QFT as string field, as can be seen by making the Klein transformation written above. Actually the stress-energy tensor always accompanies the identity operator, so the ideas must be essentially the same. Eqs. (7), (9) embody the idea that the condensate of the string field provides the kinetic part of the equation of motion. This approach is itself based on the suggestion in ref. [24] that the linear/bilinear equation of motin is the RG fixed point condition.

One idea of ref. [24] - linear gauge symme try in the interacting theory - is not followed here ${ }^{\neq 5}$. Shifting the (on-shell) background shifts the conformal operators by an expression linear in the field. It would be difficult to make the gauge invariance linear for an arbitrary background. Curiously, though, it is possible to write an extremely simple gauge invariant quadratic action functional

$S\left(\phi, \chi^{n}\right)=\left(\phi-L_{-n} \chi^{n}\right) \dagger\left(L_{0}-1\right)\left(\phi-L_{-n} \chi^{n}\right)$

employing Stuckelberg fields $\chi^{n}, n=1,2, \ldots$. The linear gauge invariance is $\phi \rightarrow \phi+L_{-n} \epsilon^{n}, \chi^{n} \rightarrow$ $\chi^{n}+\epsilon^{n}$.

Fradkin and Tseytlin [27] have proposed an approach to off-shell string theory based on Polyakov's formulation [5] of the first quantized string. The string is still described by the classical action of the general nonlinear mode, an infinite collection of tensor fields on spacetime. But the nonlinear model

\footnotetext{
\#5 Arguments against linear gauge invariance came from Shenker and Banks. Shenker pointed out that linearity would imply duality off-shell.
} 
is made to be invariant under reparame trizations by introducing the $2 \mathrm{D}$ metric as a dynamical variable. In conformal gauge for the 2D metrics, the local scale factor of the 2D metric remains a dynamical variable, to ensure that the 2D QFT of Polyakov and FradkinTseytlin is always conformally invariant, on-shell and off. Fradkin and Tseytlin's classical action for the tensor string fields is the partition function of the conformally invariant QFT on the sphere; the quantum corrections are the partition functions on surfaces of higher genus. One difficulty with this approach is that the string field interaction does not yet have the bilinear form of the joining-splitting interaction. Another difficulty is that the dynamics of the local 2D scale factor is not understood.

The basic principle underlying the approach to string theory suggested here is that the string field exactly describes the environment in which string propagates. The string fields are thus two dimensional quantum field theories. The RG equation (2) is taken as a clue that conformal invariance (duality) is exactly the on-shell condition of classical string field theory. Given that the off-shell string field lies in a larger space of conformally non-invariant QFTs, the on-shell quantum string field will not be conformally invariant ${ }^{\neq 6}$. The string field as $2 D$ QFT should not be described by its classical action, but by the-quantum stress-energy tensor and/or the quantum action functional $|0\rangle_{\xi}$. Gauge invariance of the string field theory should be associated with equivalence of $2 \mathrm{D}$ QFTs. There should be an $e f$ fective calculus of 2D QFT in which to do string field theory.

The algebraic structure of the string field theory should be the origin of locality in spacetime and of space time geometry. The algebraic origin of the string field equation of motion should explain the appearance of only bilinear interactions, which allows the dual string theory to express geometry. The commutation relations of the conformal algebra, expressed as an operator product condition on the stress-energy tensor of 2D QFT, provides a possibility.

\footnotetext{
\$6 The importance of non-scale invariant 2D QFT in string theory was emphasized by Lovelace, private conversation.
}

I am endebted to T. Banks, C. Lovelace, E. Martinec and S.H. Shenker for helpful conversations. I am grateful for the hospitality of the Racah Institute, Hebrew University of Jerusalem, Département de Physique Théorique, CEN Saclay, and the Pagosuneko Eyerra, Euskady.

\section{References}

[1] D. Friedan, Phys. Rev. Lett. 45 (1980) 1057.

[2] D. Friedan, U.C. Berkeley, Ph.D. Thesis (August 1980), LBL preprint LBL-11517 (August 1980), to be published in Ann. Phys. (September 1985).

[3] J. Scherk and J.H. Schwarz, Nucl. Phys. B81 (1974) 118; Phys. Lett. 52B (1974) 347.

[4] Y. Nambu, Proc. Intern. Conf. on Symmetries and quark models (Wayne State University, 1969); H.B. Nielsen, Proc. 15th Intern. Conf. on High-energy physics (Kiev, 1970); L. Susskind, Nuovo Cimento 69 A (1970) 457.

[5] A.M. Polyakov, Phys. Lett. 103B (1981) 207, 211; see also K. Fujikawa, Phys. Rev. D25 (1982) 2584; M. Kato and K. Ogawa, Nucl. Phys. B21 2 (1983) 433; S. Hwang, Phys. Rev. D28 (1983) 2614.

[6] D. Friedan, in: Les Houches, Session XXXIX - Recent advances in field theory and statistical mechanic, eds. J.-B. Zuber and R. Stora (North-Holland, Amsterdam, 1984).

[7] K. Bardacki, private communications (July 1983).

[8] C. Lovelace, Phys. Lett. 135B (1984) 75; and unpublished results.

[9] D. Friedan and S.H. Shenker, Aspen Center for Physics (July-August 1984), unpublished; ITP Santa Barbara (August-September 1984), unpublished; Proc. Santa Fe meeting of the APS Division of Particles and Fields (October 29-November 3, 1984), to be published.

[10] D. Friedan, Z. Qui and S.H. Shenker, Phys. Lett. 151B (1985) 37.

[11] D. Friedan, E. Martinec and S.H. Shenker, Phys. Lett. 160B (1985) 95.

[12] P. Candelas, G. Horowitz, A. Strominger and E. Witten, UCSB preprint, to be published in Nucl. Phys. B; D. Nemeschansky and S. Yankielowicz, Phys. Rev. Lett. 54 (1985) 620;

S. Jain, R. Shankar and S. Wadia, Tata Inst. preprint TIFR/TH/85-3.

[13] C. Callan, D. Friedan, E. Martinec and M. Perry, Princeton preprint (June 1985); A. Sen, Fermilab preprint (June 1985).

[14] F J. Wegner, in: Phase transitions and critical phenomena, eds. C. Domb and M.S. Green (Academic Press, New York, 1976).

[15] P. Ramond, Phys. Rev. D3 (1971) 2415;

A. Neveu and J. Schwarz, Nucl. Phys. B31 (1971) 86; F. Gliozzi, J. Scherk and D. Olive, Nucl. Phys. B122 (1977) 253. 
[16] M. Green and J. Schwarz, Nucl. Phys. B1 81 (1981) 502; Phys. Lett. 109B (1982) 444; D. Gross, J. Harvey, E. Martinec and R. Rohm, Phys. Rev. Lett. 54 (1985) 502, Princeton preprint and to be published.

[17] M.A. Bershadsky, V.G. Knizhnik and M.G. Teitelman, Phys. Lett. 151B (1985) 31.

[18] L.Alvarez-Gaumé and D.Z. Freedman, Phys. Lett. 94B (1980) 171; Phys. Rev. D22 (1980) 846.

[19] C. Hull, IAS preprints (1985);

L. Alvarez-Gaumé, S. Coleman and P. Ginsbarg, Harvard preprint (June 1985).

[20] P.A.M. Dirac, Phys. Z. Sowjetunion, Band 3, Heft 1 (1933), reprinted in: Selected papers on quantum electrodynamics, ed. J. Schwinger (Dover, New York, 1958);

D. Friedan, unpublished;

A.M. Polyakov, unpublished; and provate conversation (May 1983);
A.A. Belavin, A.M. Polyakov and A.B. Zamolodchikov, Nucl. Phys. B241 (1984) 333.

[21 ] C. Marshall and P. Ramond, Nucl. Phys. B85 (1975) 375.

[22] W. Siegel, Phys. Lett. 148B (1984) 276;149B (1984) 157, 162; 151 B (1985) 391, 396; in: Proc. ArgonneChicago Conf. on Anomalies and strings (March 28-30 1985), Berkeley preprint UCB-PTH-85/16, to be published.

[23] T. Banks and M. Peskin, in: Proc.Atgonne-Chicago Conf. on Anomalies and strings (March 28-30 1985), to be published.

[24] D. Friedan, EFI preprint 85-27 (A pril 1985).

[25] M. Kaku, City College preprint (1985).

[26] A. Neveu and P. West, Cern Preprint (July 1985).

[27] E.S. Fradkin and A.A. Tseytlin, Lebedev preprints 84261, 85-150. 